\title{
Sexual Satisfaction Is Reduced in the Female Patient and Sexually Intimate Partners Following Cancer Therapy
}

\author{
Alan M. Martinez¹, Julie M. Sroga1, Ilana B. Ressler², Leslie Ayensu-Coker, \\ Michael A. Thomas', Steven R. Lindheim4 \\ ${ }^{1}$ Department of Obstetrics \& Gynecology, University of Cincinnati, Cincinnati, USA \\ ${ }^{2}$ New York Fertility Services, New York, USA \\ ${ }^{3}$ Department of Obstetrics \& Gynecology, University of Kentucky, Lexington, USA \\ ${ }^{4}$ Department of Obstetrics \& Gynecology, Wright State University, Dayton, USA \\ Email: martinezalan@hotmail.com, srogaje@ucmail.uc.edu, Iressler@nyfsb.com, thomasma@ucmail.uc.edu, \\ doclalalindheim@yahoo.com
}

Received 22 August 2014; revised 22 September 2014; accepted 7 October 2014

Copyright (C) 2014 by authors and Scientific Research Publishing Inc.

This work is licensed under the Creative Commons Attribution International License (CC BY). http://creativecommons.org/licenses/by/4.0/

(c) (i) Open Access

\section{Abstract}

As cancer survival has continued to improve, cancer patients and their sexually intimate partner (SIP) are confronted with a number of issues including sexual function and overall sexual health. Our study objective was to assess changes in sexual function in women undergoing cancer treatment and their SIP, and attempt to identify areas of needed support and improvement. In this questionnaire-based observational study, females $(n=11)$ completed a Female Sexual Function Index (FSFI) and for SIP's (n = 11), a Brief Sexual Function Inventory (BSFI). Level of satisfaction prior to and within 3 months following treatment with surgery, chemotherapy and/or radiation was compared. Mean pre- and post-treatment total $(30.7 \pm 2.7$ vs. $23.2 \pm 3.7, \mathrm{p}<0.001)$ and individual FSFI domains were significantly different for desire $(4.2 \pm 0.6$ vs. $2.6 \pm 0.6, p<0.001)$, arousal ( $4.9 \pm 0.4$ vs. $3.5 \pm 0.7, p=0.001)$, lubrication ( $5.5 \pm 0.4$ vs. $4.5 \pm 0.8, p=0.006)$, orgasm (5.0 \pm 0.6 vs. $3.8 \pm 0.7, p=0.001$ ), satisfaction ( $5.3 \pm 0.6$ vs. $3.5 \pm 0.9, p<0.001$ ), though no differences were noted with dyspareunia. For SIP's, BSFI domain scores for level of satisfaction were significantly lower following partner treatment of SIPs (3.5 \pm 5.2 vs. $2.5 \pm 5.2, p=0.004)$. Overall, female sexual dysfunction was identified in $9 \%$ of patients prior to cancer treatment and $91 \%(\mathrm{n}=$ $11, p<0.001$ ) following treatment. In conclusion, cancer treatment significantly affects sexual function in female cancer patients and their SIPs. While long term effects on sexual dysfunction are still unclear, short-term health care strategies including treatment, counseling, and appropriate referral for cancer patients and their SIPs should not be overlooked. 


\section{Keywords}

\section{Sexuality, Sexual Dysfunction, Female Cancer, Cancer Treatment}

\section{Introduction}

Overall cancer rates in reproductive age men and women have continued to rise in recent years, Annually, more than 130,000 cancer patients are diagnosed during their reproductive years (up to age 45). Overall cancer mortality rates have declined, causing a rise in survival due to advances in early detection and more effective therapies [1]. Since 1990, the prevalence of cancer survivors in young adults has increased from 1 in 1000 to as many as 1 in 250 patients. Currently, sixty-four percent of all cancer survivors have survived 5 years or more; 40\% have survived 10 years or more; and 15\% have survived 20 years or more after diagnosis [2]. However, despite the improved treatment efficacy, a number of issues have been raised including increased prevalence of mood disorders, chronic pain, and sexual health issues in cancer survivors.

The primary focus of cancer therapy is remission or cure. With aggressive interventions, late effects of cancer therapy become evident, including a negative impact on reproductive health. Chemotherapy can impair female ovarian function resulting in infertility [3] [4] and iatrogenic menopause; radiation can create permanent tissue changes resulting in fibrosis, scarring and dyspareunia; while surgery can result in the removal of vital reproductive organs. Each can have complex effects on sexual function that relate to perception of body image, loss of sensation, feelings of lost femininity, and stability of a partnered relationship [5].

Limited research has shown that women undergoing cancer therapy are at a higher risk of sexual dysfunction including sexual desire, dyspareunia, and lubrication issues [6]-[8]. The paucity of data (focusing on the patient) is due in part because the discussion of sexuality is "taboo" and often construed as an embarrassing topic for both the patient and their SIP. The problem may be further compounded by medical providers who may not provide proper counseling and care concerning sexual health due to a lack of knowledge of current treatment options or their own personal uneasiness with this topic [9].

This study investigates the impact on overall sexual function that cancer patients and their SIPs may experience during the course of treatment using a Female Sexual Function Index (FSFI) and a Brief Sexual Function Inventory (BSFI) for SIP's prior to and within 3 months following cancer treatment. Individual FSFI domains were also evaluated assessing specific areas of sexual function including desire, arousal, lubrication, orgasm, satisfaction, and dyspareunia. We attempted to specifically identify areas of needed treatment and improvement.

\section{Materials and Methods}

This study was reviewed and approved by the University of Cincinnati College of Medicine and the Christ Hospital Institutional Review Boards (Cincinnati, $\mathrm{OH}$ ) and the study was in compliance with privacy-act guidelines.

\subsection{Patient Population}

From March 2010 and December of 2012, patients were recruited to participate in this prospective questionnaire-based observational study at the University of Cincinnati Center for Reproductive Health. Written informed consent was presented and obtained for female cancer patients presenting for oncofertility options. All women and their SIPs were eligible except those who had already received treatment for cancer.

\subsection{Study Questionnaire}

Sexual function was assessed at two points in time: baseline (pre-treatment) and within three months following completion of cancer treatment (post-treatment). Female sexual function was determined using the FSFI which is a validated multidimensional self-report instrument that addresses 6 functional domains, including desire, arousal, lubrication, orgasm, satisfaction, \& pain [10]. The FSFI provides an individual score for each domain and a total score that determines overall sexual function. A total of 19 questions are included, graded on a scale of 0 to 5, with minimum and maximum possible scores of 2 and 36, respectively. Overall female sexual dysfunction (FSD) for the FSFI questionnaire is defined as a total mean score of $<26.5$. Male sexual function was 
assessed using the BSFI, which is the first validated patient self-administered questionnaire that identifies 3 functional domains, including sexual drive, erectile function, \& ejaculatory function, and also includes assessment of perceived problem and overall sexual satisfaction. A total of 11 questions are included, graded on a scale of 0 to 4. In contrast to the FSFI, it is recommended that each individual domain is independently assessed for a change in function as opposed to an overall summary score [11] [12]. Both questionnaires are designed to be succinct and discrete, self-administered screening tools to assess sexual function.

Analysis of data was restricted to include female patients that completed both the baseline and post-treatment questionnaires. Once the questionnaire scores were obtained, paired t-tests were used to analyze changes in sexual function for both the patient and their SIP.

\section{Results}

A total of eleven women and ten SIPs who completed the FSFI at baseline and post-treatment were included for analysis. The mean age of the cancer treated women was $28.9 \pm 4.8 \mathrm{yrs}$, and SIPs was $29.5 \pm 5.5 \mathrm{yrs}$. A total of six different cancer diagnoses were seen in our study population, including five patients with breast cancer, two with Hodgkin's Lymphoma, two with central nervous system tumors, one with ovarian cancer, and one with osteosarcoma. Although our study was not exclusive to heterosexual women, all the questionnaires completed in this study group were by self-identified heterosexual women, and one female participant with a sexual partner at the time of cancer diagnosis had a change in SIP following her treatment.

Overall total mean FSFI score decreased by $24 \%$, from 30.7 ( \pm 2.7$)$ to $23.2( \pm 3.7)$ from pre to post-treatment, respectively (Figure 1). While one patient (9\%) was identified as having FSD prior to their treatment, ten of eleven women $(91 \%)$ were identified as having FSD (total score < 26.5) following treatment. With respect to individual FSFI scores, a significant decrease in five of six domains, including desire, arousal, lubrication, orgasm, and satisfaction $(\mathrm{p} \leq 0.006)$ were identified following treatment. A decrease in dyspareunia score was seen but was not significant compared to baseline ( $\mathrm{p} \leq 0.07)$ (Figure 2).

With respect to SIPs, level of satisfaction was significantly decreased from pre to post-treatment (3.5 $\pm 5.2 \mathrm{vs.}$ $2.5 \pm 5.2, \mathrm{p} \leq 0.004$ ) (Figure 3), though no individual BSFI functional domain scores including drive, erections, and ejaculation differences were noted.

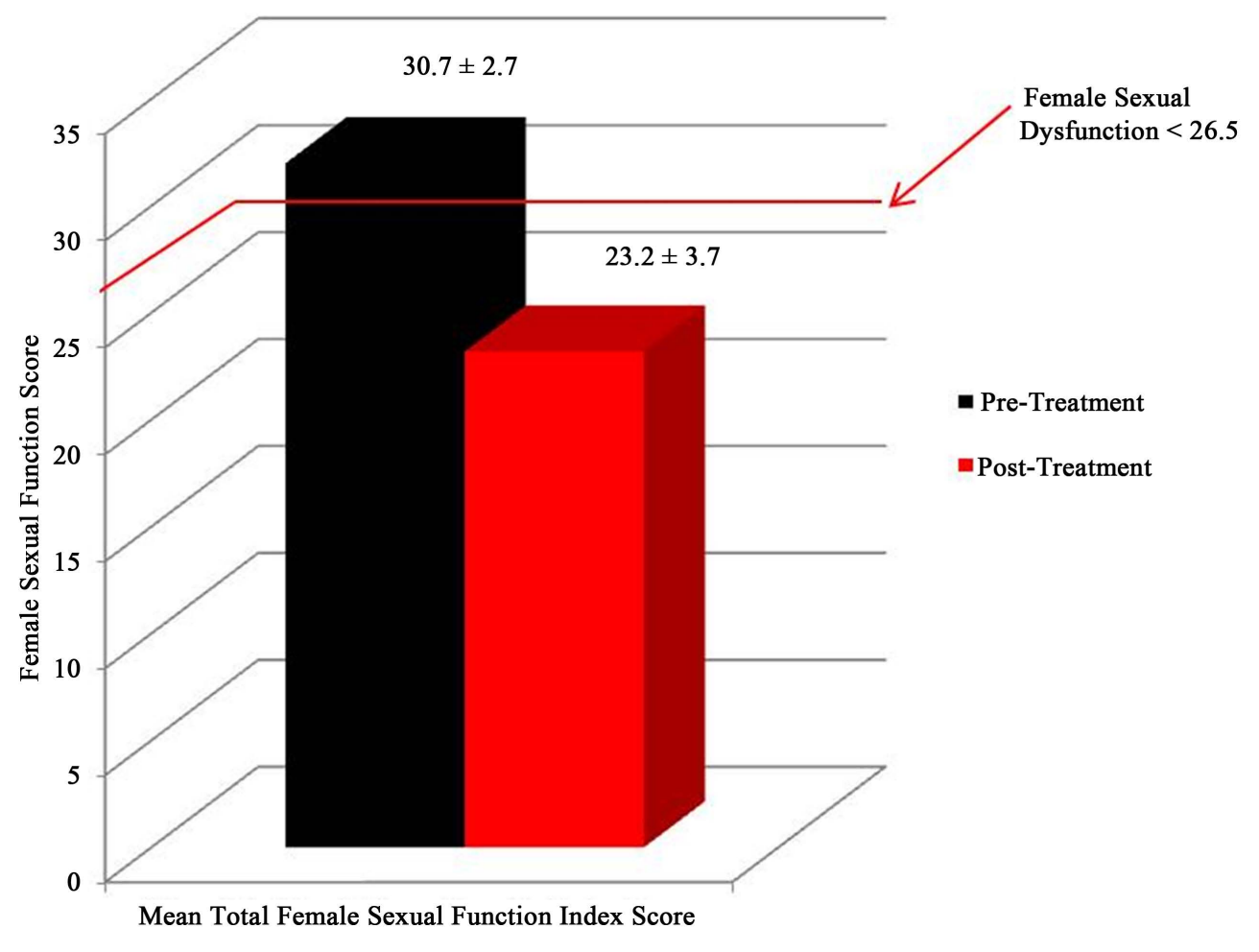

Figure 1. Comparision of total FSFI score pre and post-treatment. 


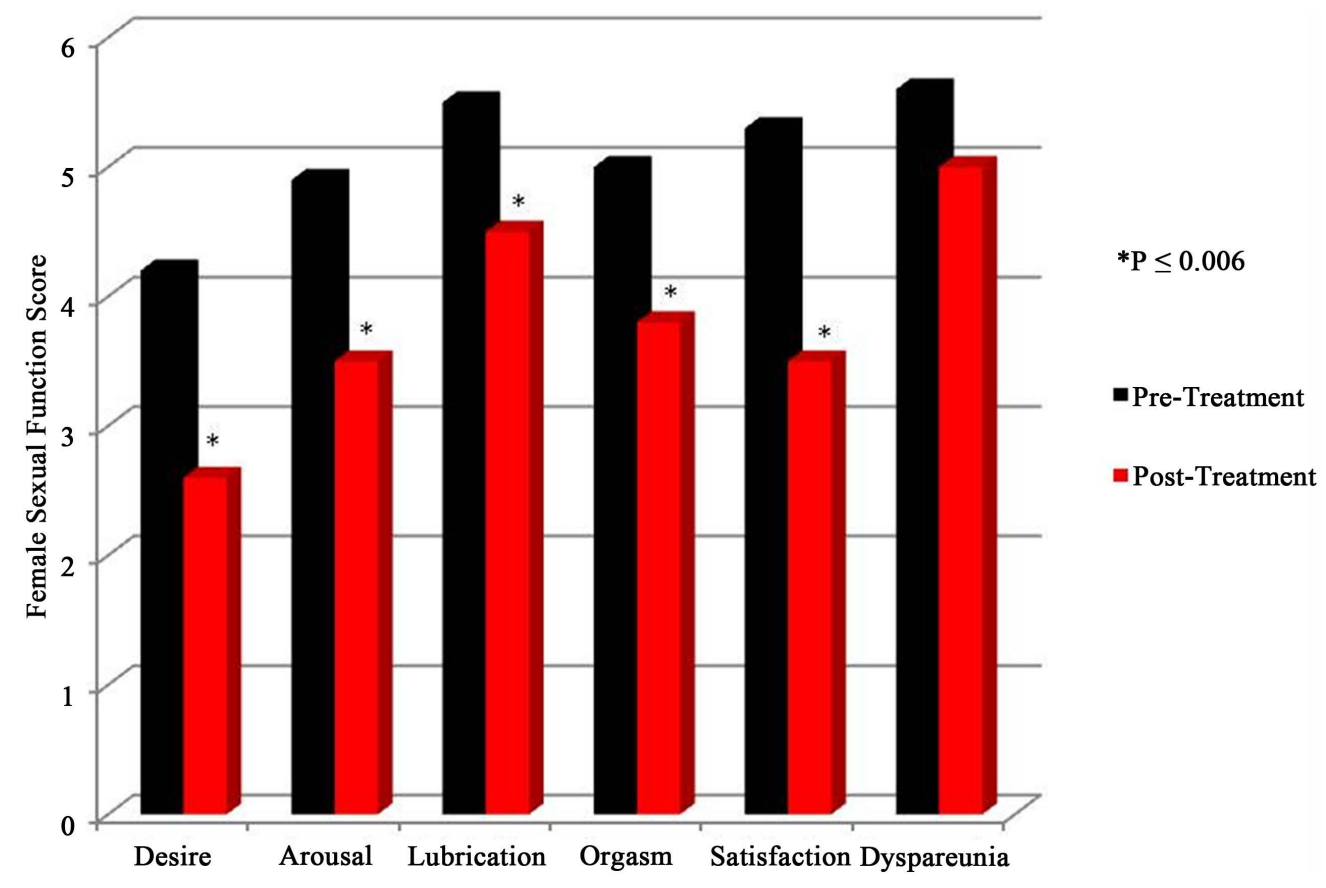

Figure 2. Comparision of individual female domain scores pre and post-treatment.

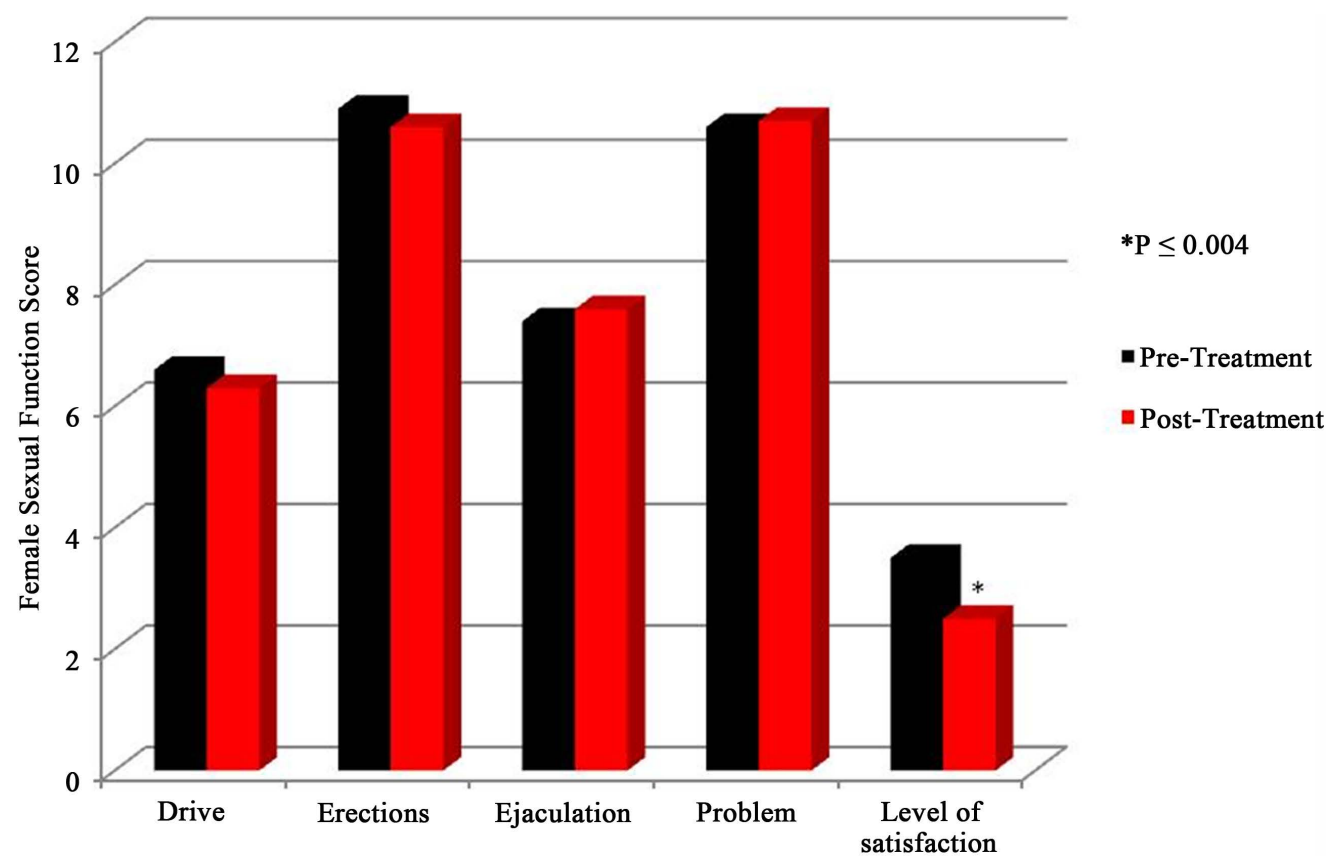

Figure 3. Comparision of male sexual function inventory scores.

\section{Discussion}

To our knowledge, this study in contrast to others objectively assesses female and male sexual dysfunction in cancer patients and their SIPs undergoing therapy using validated FSFI and BSFI questionnaires. Our data support the notion that the majority of reproductive age women undergoing cancer treatment suffer from sexual dysfunction during and following their therapy with significant effects related to desire, arousal, lubrication, orgasm, and satisfaction. Similarly, SIPs had a significant reduction in level of satisfaction, however, no adverse effects were seen related to drive, erections, and ejaculation. 
As a result of advances in cancer treatments, survival rates from cancers have dramatically increased over the past several decades, with the relative 5-year survival rate from all cancers combined (both male and female) now approximately 68\% [13]. Thus many female cancers survivors can expect to live longer lives, making previously ignored issues such as fertility preservation an important quality of life issue [14]. A multidisciplinary approach has emerged in treatment of the reproductive age woman undergoing cancer therapy involving the surgical and medical oncologist, geneticist, and reproductive endocrinology infertility specialist. This is critical as many patients are fearful that their history of cancer or its treatment will adversely impact their fertility and potentially increase their risk of recurrence. The psychological impact of cancer is profound with many survivors suffering from stress, grief, and depression [15] [16]. Therefore, it is highly crucial for health care providers to appropriately discuss the impact of cancer on reproductive health.

A heightened awareness has occurred in the past 10 years not just to the physical effects of cancer treatment, but also on the impact on future fertility where the efforts of the American Society of Clinical Oncology (ASCO) and the American Society of Reproductive Medicine (ASRM) have formally recognized fertility awareness in the treatment of reproductive age women undergoing cancer therapy [14] [17]. However, other issues related to reproductive health and sexual function due to debilitating and distorting surgical and radiation effects, and hypoestrogenemia secondary to iatrogenic menopause are often overlooked. These include perception of body image, loss of sensation, feelings of lost femininity, and stability of a partnered relationship [5].

Limited research has shown that women undergoing cancer therapy are at a higher risk of sexual dysfunction. Although certain chemotherapy-associated toxicities including nausea and emesis exist, progress has been made in reducing these effects. However, the emotional, social, and sexual consequences of cancer treatment present continuing challenges in efforts to optimize quality of life [18]. A study of 752 cancer survivors found a $41 \%$ rate of sexual dysfunction 1 year following treatment [19]. The female participants in this study reported a larger number of psychological and physical problems, and younger cancer patients (ages 18 - 54 yrs) were found to have the largest incidence of sexual dysfunction following treatment. A 2010 review on breast cancer research demonstrated that sexual changes are often the most problematic aspect of a woman's life following breast cancer, and supported the idea of complex deficiencies in sexuality following breast cancer [20]. It appears that decreased sexual functioning and quality of life for female cancer patients are greatest during the first year following diagnosis, however in some women there is evidence that persistent dysfunction may persist [10] [18] [20].

The results of our study objectively supports that female patients undergoing cancer treatment will suffer from sexual dysfunction related to desire, arousal, lubrication, orgasm, and satisfaction during and after their course of treatment. We acknowledge there are limitations in our study including the small sample size and failure to categorize FSD based on cancer type and type of cancer treatment. The self-reported responses to the FSFI and BSFI questionnaires rely on patient recollection; therefore attempts to administer the standardized questionnaires at consistent time points prior to and following treatment were followed to limit any self-reporting biases. The strength of our study lies in the objective assessment sexual dysfunction and assessment of SIPs and underscores the importance of provider discussion of 1) sexual function in general to both the patient and their SIP 2) the possible adverse impact on FSD from cancer therapies and 3) appropriate referral emphasizing the importance of a social worker, psychologist, and a sex therapist as part of the multi-discipline team approach to cancer patients and their SIPs. Some reassuring data suggests that the most consistent predictor of satisfying sexual function is the quality of their relationship [6] [20].

Further work in this area is clearly needed and should investigate sexual dysfunction over a continuum of time among specific cancer types and treatment regimens. This would allow characterization of specific sexual dysfunction with regards to individual cancer types, and perhaps tailor counseling and treatment.

\section{Conclusion}

Our study demonstrates a reduction in sexual function for female cancer patients and their SIPs following cancer treatment. Timely treatment, counseling, and appropriate referral for sexual dysfunction should not be neglected or overlooked in physicians caring for these patients.

\section{Acknowledgements}

Support: Patty Brisben Foundation for Women’s Sexual Health. 


\section{References}

[1] Figueroa-Moseley, C., et al. (2007) Behavioral Interventions in Treating Anticipatory Nausea and Vomiting. Journal of the National Comprehensive Cancer Network, 5, 44-50.

[2] de Moor, J.S., et al. (2013) Cancer Survivors in the United States: Prevalence across the Survivorship Trajectory and Implications for Care. Cancer Epidemiology, Biomarkers Prevention, 22, 561-570. http://dx.doi.org/10.1158/1055-9965.EPI-12-1356

[3] Stead, M.L., et al. (2007) Psychosexual Function and Impact of Gynaecological Cancer. Best Practice Research Clinical Obstetrics Gynaecology, 21, 309-320. http://dx.doi.org/10.1016/j.bpobgyn.2006.11.008

[4] Carelle, N., et al. (2002) Changing Patient Perceptions of the Side Effects of Cancer Chemotherapy. Cancer, 95, 155163. http://dx.doi.org/10.1002/cncr.10630

[5] Gilbert, E., Ussher, J.M. and Perz, J. (2010) Sexuality after Breast Cancer: A Review. Maturitas, 66, 397-407. http://dx.doi.org/10.1016/j.maturitas.2010.03.027

[6] Archibald, S., Lemieux, S., Byers, E.S., Tamlyn, K. and Worth, J. (2006) Chemically-Induced Menopause and the Sexual Functioning of Breast Cancer Survivors. Women and Therapy, 29, 83-106. http://dx.doi.org/10.1300/J015v29n01_05

[7] Ganz, P.A., et al. (1998) Life after Breast Cancer: Understanding Women's Health-Related Quality of Life and Sexual Functioning. Journal of Clinical Oncology, 16, 501-514.

[8] Barni, S. and Mondin, R. (1997) Sexual Dysfunction in Treated Breast Cancer Patients. Annals of Oncology, 8, 149153. http://dx.doi.org/10.1023/A:1008298615272

[9] Hautamaki, K., et al. (2007) Opening Communication with Cancer Patients about Sexuality-Related Issues. Cancer Nursing, 30, 399-404. http://dx.doi.org/10.1097/01.NCC.0000290808.84076.97

[10] Rosen, R., et al. (2000) The Female Sexual Function Index (FSFI): A Multidimensional Self-Report Instrument for the Assessment of Female Sexual Function. Journal of Sex Marital Therapy, 26, 191-208. http://dx.doi.org/10.1080/009262300278597

[11] O’Leary, M.P., Fowler, F.J., Lenderking, W.R., Barber, B., Sagnier, P.P., Guess, H.A., et al. (1995) A Brief Male Sexual Function Inventory for Urology. Urology, 46, 697-706. http://dx.doi.org/10.1016/S0090-4295(99)80304-5

[12] Rosen, R.C. (1996) Brief Male Sexual Function Inventory for Urology. Urology, 47, 782-783. http://dx.doi.org/10.1016/S0090-4295(96)00075-1

[13] Jemal, A., Thun, M.J., Ries, L.A.G., Howe, H.L., Weir, H.K., Center, M.M., et al. (2008) Annual Report to the Nation on the Status of Cancer, 1975-2005, Featuring Trends in Lung Cancer, Tobacco Use, and Tobacco Control. Journal of the National Cancer Institute, 100, 1672-1694. http://dx.doi.org/10.1093/jnci/djn389

[14] Lee, S.J., Schover, L.R., Partridge, A.H., Patrizio, P., Wallace, W.H., Hagerty, K., et al. (2006) American Society of Clinical Oncology Recommendations on Fertility Preservation in Cancer Patients. Journal of Clinical Oncology, 24, 2917-2931. http://dx.doi.org/10.1200/JCO.2006.06.5888

[15] Schover, L.R., Rybicki, L.A., Martin, B.A. and Bringelsen, K.A. (1999) Having Children after Cancer. A Pilot Survey of Survivors' Attitudes and Experiences. Cancer, 86, 697-709.

[16] Skinner, R., Wallace, W.H. and Levitt, G.A. (2006) Long-Term Follow-Up of People Who Have Survived Cancer during Childhood. The Lancet Oncology, 7, 489-498. http://dx.doi.org/10.1016/S1470-2045(06)70724-0

[17] The Ethics Committee of the American Society for Reproductive Medicine (2005) Fertility Preservation and Reproduction in Cancer Patients. Fertility and Sterility, 83, 1622-1628. http://dx.doi.org/10.1016/j.fertnstert.2005.03.013

[18] Shimozuma, K., Ganz, P.A., Petersen, L. and Hirji, K. (1999) Quality of Life in the First Year after Breast Cancer Surgery: Rehabilitation Needs and Patterns of Recovery. Breast Cancer Research and Treatment, 56, 45-57. http://dx.doi.org/10.1023/A:1006214830854

[19] Baker, F., Denniston, M., Smith, T. and West, M.M. (2005) Adult Cancer Survivors: How Are They Faring? Cancer, 104, 2565-2576. http://dx.doi.org/10.1002/cncr.21488

[20] Ganz, P.A., Desmond, K.A., Belin, T.R., Meyerowitz, B.E. and Rowland, J.H. (1999) Predictors of Sexual Health in Women after a Breast Cancer Diagnosis. Journal of Clinical Oncology, 17, 2371-2380. 
Scientific Research Publishing (SCIRP) is one of the largest Open Access journal publishers. It is currently publishing more than 200 open access, online, peer-reviewed journals covering a wide range of academic disciplines. SCIRP serves the worldwide academic communities and contributes to the progress and application of science with its publication.

Other selected journals from SCIRP are listed as below. Submit your manuscript to us via either submit@scirp.org or Online Submission Portal.
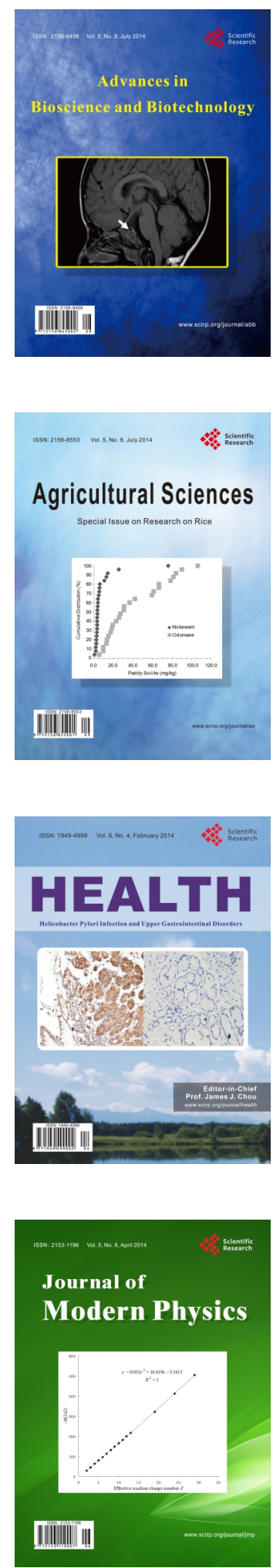
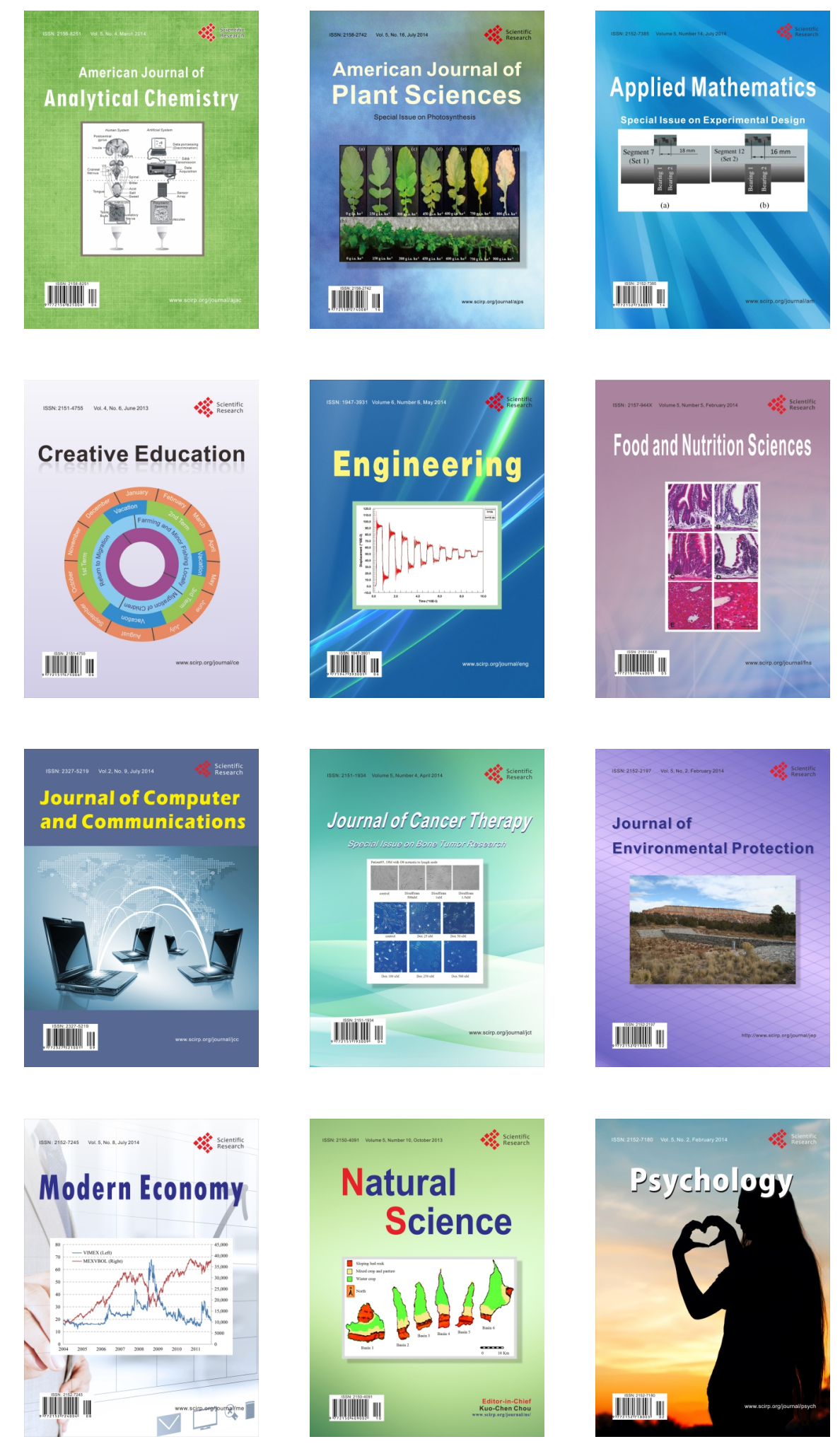